\title{
Characteristics of hospitalised patients with 2009 H1N1 influenza in Chile
}

\author{
R. Riquelme*, M. Riquelme*, M.L. Rioseco*, C. Inzunza*, Y. Gomez*, C. Contreras*, \\ J. Riquelme*, P. Peyrani ${ }^{\#}$, T. Wiemken ${ }^{\#}$ and J. Ramirez ${ }^{\#}$
}

ABSTRACT: As the pandemic of $2009 \mathrm{H} 1 \mathrm{~N} 1$ influenza A virus progressed, more patients required hospitalisation. The objective of this study is to describe the characteristics and clinical course of hospitalised patients with 2009 H1N1 virus infection in Chile.

This was a prospective, observational study of 100 consecutive hospitalised patients with RTPCR-confirmed 2009 H1N1 influenza A, admitted to Puerto Montt General Hospital (Puerto Montt, Chile). Information was obtained regarding contact history, demographics, laboratory values and clinical course.

The primary reason for hospitalisation was pneumonia, in $75 \%$ of patients. Rapid influenza A test was positive in $51 \%$ of patients. Prior exposure to $2009 \mathrm{H} 1 \mathrm{~N} 1$ virus was documented in $21 \%$ of patients. Clinical failure, documented in $18 \%$ of cases, was characterised by respiratory failure and acute respiratory distress syndrome. Failure was more common in patients with obesity, tachypnoea, confusion and multilobar infiltrates.

When evaluating a patient hospitalised with influenza-like illness, a negative rapid test for influenza A or negative contact with a suspected case should not alter physicians' considerations regarding the likelihood of 2009 H1N1 virus infection. Patients with 2009 H1N1 virus infection with obesity, tachypnoea, confusion and multilobar infiltrates should be closely monitored since they are at high risk for clinical failure.

KEYWORDS: Chile, H1N1, influenza, outcomes, pneumonia, swine flu

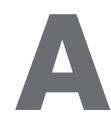
novel influenza A virus (2009 H1N1) was identified as the cause of an outbreak of respiratory illness in Mexico in March 2009 [1]. The 2009 H1N1 virus rapidly spread to several countries, and in July 2009, the World Health Organization (WHO) declared that infections due to 2009 H1N1 virus had reached pandemic level [2]. Although most reported cases were mild, the number of young patients hospitalised continued to rise [3, 4].

A limited number of observational studies have reported the clinical and laboratory characteristics of hospitalised patients with 2009 H1N1 virus infection, with the majority of reported cases being patients with severe disease requiring intensive care unit (ICU) admission [5-10]. Physicians were confronted with many patients requiring hospitalisation due to severe 2009 H1N1 virus infection at a time that knowledge regarding initial presentation, clinical course and management strategies was limited.

Corresponding with the start of influenza season in the southern hemisphere, the country of Chile reported its first case of 2009 H1N1 influenza in
mid-May 2009. The virus rapidly spread throughout the country and by the end of May, cases had been reported in 11 of the 15 administrative regions [11]. Puerto Montt General Hospital serves the adult population of the city of Puerto Montt, the capital of the administrative region of Los Lagos (Chile). The first case of 2009 H1N1 influenza virus in the city of Puerto Montt was detected on May 24, 2009. In order to contribute to the cases described in the literature and to answer some of the clinical questions regarding this disease, we planned a prospective study to describe the clinical and laboratory characteristics of 100 consecutive patients with 2009 H1N1 influenza virus infection hospitalised at the Puerto Montt General Hospital.

\section{METHODS}

\section{Study design and patients}

This was a prospective, observational study of consecutive adult patients hospitalised with an influenza-like illness at the Puerto Montt Hospital from May 29-July 7 2009. The local institutional review board approved conduct of the study without consent from participants. All patients had a complete history, and physical and
AFFILIATIONS

*Puerto Montt Hospital, Puerto Montt, Chile.

\#Division of Infectious Diseases, University of Louisville, Louisville, KY, USA.

CORRESPONDENCE

J. Ramirez

Division of Infectious Diseases

501 East Broadway \#380

University of Louisville

Louisville

KY 40202

USA

E-mail: j.ramirez@louisville.edu

Received:

Nov 122009

Accepted after revision:

Feb 042010

First published online:

Feb 252010 
laboratory evaluation at the time of hospital admission. Patients were evaluated by a study investigator with a subspecialty in pulmonary medicine, during each day of hospitalisation. Since this study occurred during the midst of a national epidemic, once the emergency room physician indicated that a patient with an influenza-like illness required hospitalisation, the patient was evaluated by a member of the pulmonary division, regardless of the severity of disease. Respiratory samples were obtained for rapid immunofluoresence influenza test for detection of influenza A antigens, as well as for RT-PCR for detection of 2009 H1N1 virus. All RTPCR tests were performed at the National Institute for Public Health, (Santiago, Chile). The extent of microbiological workup was decided by the primary treating physician. The local pneumonia pathway recommended empiric $\beta$-lactam monotherapy for mild disease or combination therapy with a $\beta$ lactam plus a quinolone or macrolide for severe disease. After the results of RT-PCR were available, patients with negative RT-PCR for 2009 H1N1 influenza A virus were excluded from the study.

\section{Study definitions}

Influenza-like illness

A patient was considered to have an influenza-like illness when two or more of the following criteria were present at time of hospital admission: fever, chills, myalgia, rhinitis, headache, cough, wheezing, painful swallowing, abdominal pain, or diarrhoea. The day that the patient developed one of the signs and symptoms of an influenza-like illness was defined as the day of onset of disease.

\section{H1N1 virus infection}

A patient presenting with an influenza-like illness and a respiratory sample positive for 2009 H1N1 virus by RT-PCR.

\section{H1N1 virus pneumonia}

A patient fulfilling criteria for 2009 H1N1 virus infection with evidence of a new pulmonary infiltrate on chest radiography without a clear alternative diagnosis. The evaluation of the chest radiograph, performed by the investigators, was incorporated into the database.

\section{H1N1 virus pneumonia with bacterial co-infection}

A patient fulfilling criteria for 2009 H1N1 virus pneumonia with a culture positive for a bacterial pathogen from a blood sample at time of hospitalisation.

\section{Failure of outpatient therapy}

A patient who received $\geqslant 48 \mathrm{~h}$ of antiviral therapy before hospitalisation.

\section{Obesity}

A patient was considered obese if his or her body mass index was $\geqslant 30 \mathrm{~kg} \cdot \mathrm{m}^{-2}$.

\section{H1N1 virus-induced airway hyperactivity}

A patient was considered to have 2009 H1N1 virus-induced airway hyperactivity if he or she had clinical evidence of bronchospasm, without prior history of asthma or bronchial hyperactivity.

\section{Exposure to 2009 H1N1 virus}

A standardised exposure questionnaire was given to the patient or an immediate relative. According to the history during the 7 days prior to initiation of symptoms, exposure was defined as follows. 1) Definitive 2009 H1N1 virus exposure. The patient was exposed to a confirmed case of 2009 H1N1 virus infection. 2) Probable 2009 H1N1 virus exposure. The patient was exposed to a patient with an influenza-like illness. 3) Negative 2009 H1N1 virus exposure. The patient was not exposed to a patient with an influenza-like illness at the time of contact. Follow-up clinical data of the contact to evaluate future development of influenza-like illness was not obtained.

\section{Reason for hospitalisation}

According to the primary medical problem, the reasons for hospital admission were classified as follows. 1) Hospitalisation due to severe 2009 H1N1 influenza infection. 2) Hospitalisation due to deterioration of comorbidities secondary to 2009 H1N1 influenza infection. 3) Hospitalisation due to $2009 \mathrm{H} 1 \mathrm{~N} 1$ influenza complicated with pneumonia.

\section{Appropriate 2009 H1N1 virus treatment}

A patient who received oseltamivir or zanamivir at the time of hospital admission.

\section{Clinical course}

The patients' clinical course was classified as clinical failure or clinical success according to the following definitions. 1) Clinical failure. A patient was defined as having clinical failure if any of the following criteria were present: respiratory failure with need for mechanical ventilation; cardiovascular failure with need for vasopressors; and death during hospitalisation. Clinical failure was subclassified as early in patients who meet criteria within the first $72 \mathrm{~h}$ of hospital admission or late in patients who meet criteria after $72 \mathrm{~h}$ after hospital admission. 2) Clinical Success. A patient who did not meet any of the criteria for clinical failure.

\section{Statistical analysis}

Patients were divided into two groups: group 1, clinical success; and group 2, clinical failure. Baseline clinical and laboratory characteristics of the two groups were compared using Chi-squared or Fisher's Exact tests for categorical variables and Mann-Whitney U-tests for continuous variables. p-values of $\leqslant 0.05$ were considered statistically significant. SAS v9.2 (SAS Inc., Cary, NC, USA) was used for all analyses.

\section{RESULTS}

\section{Study patients}

Data from 100 consecutive patients with RT-PCR-confirmed 2009 H1N1 virus infection were analysed. A rapid immunofluoresence influenza test at time of hospitalisation was positive in $51 \%$ of patients. Prior seasonal influenza vaccination was documented in $20 \%$ of patients. Failure of outpatient therapy was documented in $17 \%$ of patients.

\section{Exposure history}

Definitive exposure was documented in $4 \%$ of patients, probable exposure in $17 \%$ of patients, and a negative exposure in $79 \%$ of patients. 


\section{Reason for hospitalisation}

2009 H1N1 influenza virus complicated with pneumonia was the reason for hospitalisation in 75\% of patients. 2009 H1N1 viral pneumonia with bacterial coinfection was not documented in any of the 25 patients in whom blood cultures were obtained. Deterioration of comorbidities was the reason for hospitalisation in $18 \%$ of patients, and $2009 \mathrm{H} 1 \mathrm{~N} 1$ influenza with significant vital sign abnormalities was the reason in $7 \%$ of patients.

\section{Antiviral treatment}

At the time of hospitalisation, appropriate antiviral therapy was given to $97 \%$ of patients. Oseltamivir was used in $91 \%$ of patients and zanamivir was used in six pregnant patients.

\section{Antibiotic treatment}

Empiric antibiotic therapy was given to $86 \%$ of the study population. Monotherapy with ceftriaxone was given to $42 \%$ of the population and combination therapy with addition of intravenous ciprofloxacin or clarithromycin was given to $46 \%$ of the study population.

\section{Clinical course}

During hospital follow-up, clinical failure was documented in $18 \%$ of patients. All patients who failed developed respiratory failure with the need for mechanical ventilation. Acute respiratory distress syndrome (ARDS) was documented in $13 \%$ of patients. Nine patients died. Clinical and laboratory characteristics comparing patients with clinical success versus clinical failure are shown in table 1. Clinical failure was not associated with any of the signs and symptoms of influenza. Clinical failure was significantly associated with signs and symptoms of respiratory compromise as well as altered mental status and obesity. Patient characteristics for the 22 patients admitted to the ICU are described in table 2.

The mean \pm SD number of days from onset of influenza-like signs and symptoms to initiation of antiviral therapy was an average of $4.6 \pm 2.5$ days for the patients with clinical failure and $4.2 \pm 2.9$ days for the patients with clinical success. The number of days from onset of influenza-like signs and symptoms prior to hospital admission was $4.7 \pm 2.8$ days for the patients with clinical failure and $4.7 \pm 3.1$ days for the patients with clinical success.

\section{DISCUSSION}

The primary findings of our study are that, in patients with 2009 H1N1 virus infection requiring hospitalisation, a rapid influenza A test identifies only $50 \%$ of infected patients, the majority of hospitalised patients have a negative exposure history, and influenza pneumonia is the primary reason for hospitalisation. Regarding the clinical course of hospitalised patients, our study indicates that even with appropriate antiviral therapy, $\sim 20 \%$ of patients will develop clinical failure. Patients with obesity, tachypnoea, confusion and multilobar infiltrates are at high risk for clinical failure.

Need for admission to an ICU was documented in $22 \%$ of the study population. In a recent publication from the USA of 272 hospitalised patients with 2009 H1N1 influenza, the need for ICU admission was documented in $25 \%$ of patients [4]. Severity of disease at the time of ICU admission for our population, evaluated by Acute Physiology and Chronic Health Evaluation (APACHE) II score (mean 15), was similar to the reported scores from several ICUs in Spain [8]. Need for invasive mechanical ventilation, documented in approximately two-thirds of patients admitted to the ICU, was also in concordance with prior published data [8].

A Centers for Disease Control and Prevention (CDC) evaluation indicated that $\sim 40-60 \%$ of $2009 \mathrm{H} 1 \mathrm{~N} 1$ virus isolates were not detected by rapid influenza tests [12]. The CDC showed that the sensitivity of rapid influenza tests increased in specimens containing high levels of 2009 H1N1 virus, but the sensitivity declined substantially as virus levels decreased. Data from patients infected with H5N1 influenza virus demonstrated a correlation of viral load with clinical outcomes [13]. Data on viral load in patients with $2009 \mathrm{H} 1 \mathrm{~N} 1$ influenza is not available but we speculated that patients with severe 2009 H1N1 virus infection, requiring hospital admission, were likely to have high viral load in respiratory secretions, and that rapid influenza tests would perform better in this selected population. Data from our study, however, indicated the same low sensitivity for the rapid influenza test, even in patients with severe 2009 H1N1 virus infection. The low sensitivity of the rapid tests has important implications for physicians evaluating patients with severe influenza-like illness. A negative rapid test does not rule out severe 2009 H1N1 virus infection, and the decision to start antiviral therapy cannot be influenced by a negative rapid influenza test. Hospitalised patients with 2009 H1N1 influenza should be placed in respiratory isolation to prevent transmission to other patients or healthcare workers. A negative rapid influenza A test should not be used to define the need for respiratory isolation in a patient suspected of having 2009 H1N1 influenza virus infection.

The Ministry of Health from the country of Chile reported a $2.4 \%$ hospitalisation rate for patients with $2009 \mathrm{H} 1 \mathrm{~N} 1$ influenza infection [14]. In the present report, we describe 100 confirmed hospitalised patients from the region of Los Lagos (Chile), where an estimated 4,167 patients were infected. 2009 H1N1 virus infection complicated with pneumonia was the most common indication for hospitalisation. Failure of outpatient therapy was documented in $17 \%$ of patients, with a full duration of therapy being completed by two patients. Our data suggest that during the initial evaluation of a patient with severe influenza-like illness, current antiviral therapy with oseltamivir does not rule out the possibility of severe $2009 \mathrm{H} 1 \mathrm{~N} 1$ virus infection. Since viral cultures and susceptibilities were not performed, we cannot exclude the possibility of antiviral resistance as an explanation of failure of outpatient therapy.

The literature from prior pandemics indicates that $30-50 \%$ of infected patients may be asymptomatic [15]. The data suggests that a significant number of infected patients would have a negative history of contact with a symptomatic case. Our study also indicates that a known history of contact with a definitive or suspected case of $2009 \mathrm{H} 1 \mathrm{~N} 1$ virus infection is infrequent in hospitalised patients with 2009 H1N1 virus infection. Since we did not have follow-up data on potential contacts, we were unable to confirm whether patients were exposed to newly infected individuals who were in the incubation period of the disease at the time of contact. When evaluating a patient with influenza-like illness, the lack of an epidemiological link 
TABLE 1 Characteristics of patients with clinical success versus clinical failure

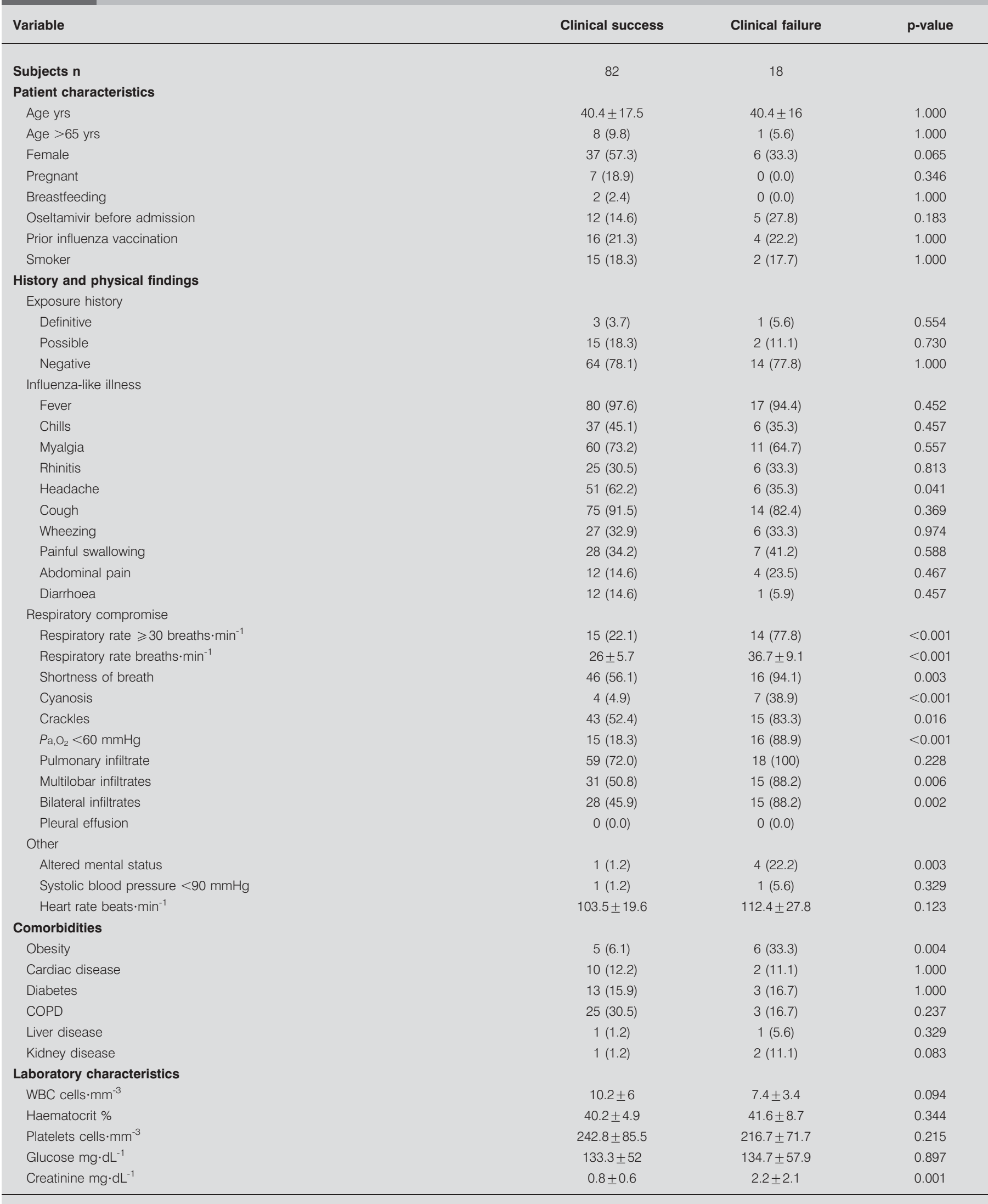

Data are presented as mean $\pm \mathrm{SD}$ or $\mathrm{n}(\%)$, unless otherwise stated. $\mathrm{Pa}, \mathrm{O}_{2}$ : arterial oxygen tension; COPD: chronic obstructive pulmonary disease; WBC: white blood cells. 


\begin{tabular}{lc}
\hline TABLE 2 & $\begin{array}{c}\text { Characteristics of patients admitted to the } \\
\text { intensive care unit (ICU) }\end{array}$ \\
\hline APACHE II score & $15.5 \pm 6.5$ \\
Glasgow Coma score $<\mathbf{1 5}$ & $5(22)$ \\
SAPS II & $28.4 \pm 12.4$ \\
Sepsis & $5(22)$ \\
Shock & $4(18)$ \\
ARDS & $13(59)$ \\
Mechanical ventilation & $15(68)$ \\
Days on mechanical ventilation & $9.5 \pm 12.6$ \\
ICU days & $10.7 \pm 12.0$ \\
ECMO & $3(14)$ \\
\hline
\end{tabular}

Data are presented as mean \pm SD and $n(\%)$. APACHE: Acute Physiology and Chronic Health; SAPS: simplified acute physiology score; ARDS: acute respiratory distress syndrome; ECMO: extracorporeal membrane oxygenation. ${ }^{\#}: \mathrm{n}=22$. ": shock was defined as systolic blood pressure $<90 \mathrm{mmHg}$ or mean arterial pressure $<60 \mathrm{mmHg}$.

should not alter physicians' concern for 2009 H1N1 virus infection. We speculate that in the pandemic of $2009 \mathrm{H} 1 \mathrm{~N} 1$ virus infection, a significant number of patients may have had subclinical disease. This would explain the high number of infected patients who required hospitalisation and had a negative exposure history.

Although most patients were treated with appropriate antiviral therapy at the time of hospital admission, clinical failure was documented in $18 \%$ of patients. It has been suggested that delayed initiation of antiviral therapy may lead to worsening of patient outcomes. In our study, there were no statistically significant differences in duration of signs and symptoms of influenza before hospitalisation or before initiation of antiviral therapy for patients with clinical success versus clinical failure. Lack of benefit of antiviral therapy in our population may be explained by a delayed initiation of therapy, since in our population, therapy commenced $\sim 4$ days after onset of signs and symptoms.

Our study failed to demonstrate an association of any of the influenza-like symptoms with clinical failure. Although pregnant females were over-represented in our study population, with a rate of $16.3 \%$, we were not able to demonstrate any association of pregnancy with poor outcomes. As the safety of oseltamivir during pregnancy was unclear at the initiation of the study period, the initial hospital policy was to treat hospitalised, pregnant females with zanamivir. Six of the seven pregnant patients in this study were treated with zanamivir.

We identified a significant association between multiple clinical and laboratory characteristics indicative of respiratory compromise with clinical failure. Obesity and altered mental status were also associated with clinical failure. Altered mental status has been reported to be associated with poor outcomes in patients with bacterial pneumonia. Obesity is not a risk factor for poor outcomes in patients with seasonal influenza, but obesity has been suggested as a risk factor for poor outcomes in patients with $2009 \mathrm{H} 1 \mathrm{~N} 1$ influenza infection in the USA [7]. Our data indicates that obesity is a risk factor for poor outcomes, even in a country where the prevalence of obesity is much lower than in the USA $[16,17]$.
Bacterial pneumonia in association with influenza has been considered an important factor leading to poor patient outcomes in prior pandemics [18]. Even though none of the blood cultures were positive, we were unable to evaluate the effect of bacterial co-infection on patient outcomes, since blood cultures were obtained in only $25 \%$ of the study population and workup for atypical pathogens was not performed. Although bacterial coinfection was not documented, the majority of the study population was treated with antibiotics according to local guidelines. Prior publications failed to demonstrate any significant involvement of bacterial pathogens in hospitalised patients with 2009 H1N1 virus pneumonia [5-10]. Future studies are necessary to define the best treatment of 2009 H1N1 virus pneumonia and the role of combination antiviral therapy. Studies are also necessary to define the efficacy of steroids or other drugs targeting the cytokine cascade in the prevention of 2009 H1N1 virus-induced ARDS.

Limitations of this study include that our data represent the experience of a single centre, and that data were collected at the time of a local outbreak of $2009 \mathrm{H} 1 \mathrm{~N} 1$ virus infection. The city of Puerto Montt, located in the south of Chile, reported more cases of hospitalised patients than cities located in the north of the country. These data are compatible with the known influence of lower temperatures in the transmission of influenza.

In conclusion, data from our study have several clinical implications. First, a negative rapid influenza A test cannot be used to rule out $2009 \mathrm{H} 1 \mathrm{~N} 1$ virus infection, even in patients with severe disease. Secondly, exposure history is of limited value, since during the pandemic, the majority of hospitalised patients were likely to have reported a negative exposure history. Thirdly, even with appropriate antiviral therapy, clinical failure may have developed in $\sim 20 \%$ of patients. Finally, patients with 2009 H1N1 virus infection that are obese, have tachypnoea, confusion, or multilobar infiltrates on chest radiograph should be closely monitored since they are at high risk for clinical failure.

\section{STATEMENT OF INTEREST}

None declared.

\section{ACKNOWLEDGEMENTS}

We acknowledge E. Smigielski (University of Louisville, Louisville, KY, USA) for her editorial and librarian support.

\section{REFERENCES}

1 Novel Swine-Origin Influenza A (H1N1) Virus Investigation Team. Emergence of a novel swine-origin influenza A (H1N1) virus in humans. N Engl J Med 2009; 360: 2605-2615.

2 World Health Organization. Transcript of statement by Margaret Chan, Director General of the World Health Organization. www. who.int/mediacentre/influenzaAH1N1_presstranscript_20090611. pdf Date last accessed: August 3, 2010. Date last updated: June 11, 2009.

3 World Health Organization. Pandemic (H1N1) 2009 www.who. int/csr/don/2009_08_04/en/index.html Date last accessed: August 3, 2010. Date last updated: July 31, 2009.

4 Jain S, Kamimoto L, Bramley AM, et al. Hospitalized patients with 2009 H1N1 influenza in the United States, April-June 2009. N Engl J Med 2009; 361: 1935-1944. 
5 Perez-Padilla R, de la Rosa-Zamboni D, Ponce de Leon S, et al. Pneumonia and respiratory failure from swine-origin influenza A (H1N1) in Mexico. N Engl J Med 2009; 361: 680-689.

6 Centers for Disease Control and Prevention. Hospitalized patients with novel influenza A (H1N1) virus infection-California, AprilMay 2009. MMWR Morb Mortal Wkly Rep 2009; 58: 470-472.

7 Centers for Disease Control and Prevention. Intensive-care patients with severe novel influenza A (H1N1) virus infection-Michigan, June 2009. MMWR Morb Mortal Wkly Rep 2009; 58: 749-752.

8 Rello J, Rodríguez A, Ibañez $\mathrm{P}$, et al. Intensive care adult patients with severe respiratory failure caused by influenza A (H1N1)v in Spain. Crit Care 2009; 13: R148.

9 Kumar A, Zarychanski R, Pinto R, et al. Critically ill patients with 2009 influenza A (H1N1) infection in Canada. JAMA 2009; 302: 1872-1879.

10 Domínguez-Cherit G, Lapinsky SE, Macias AE, et al. Critically ill patients with 2009 influenza a (H1N1) infection in Mexico. JAMA 2009; 302: 1880-1887.

11 Chile Ministry of Health. Informes diarios de prensa sobre Influenza A (H1N1) en Chile, Mayo-Junio 2009 [Daily situation updates, 26 April May-5 June, 2009]. www.pandemia.cl/ Date last accessed: November 4, 2009. Date last updated: June 5, 2009.

12 Centers for Disease Control and Prevention. Evaluation of rapid influenza diagnostic tests for detection of novel influenza A
(H1N1) virus - United States, 2009. MMWR Morb Mortal Wkly Rep 2009; 58: 826-829.

13 de Jong MD, Tran TT, Truong HK, et al. Oseltamivir resistance during treatment of influenza A (H5N1) infection. N Engl J Med 2005; 353: 2667-2672.

14 Chile Ministry of Health. Informe situacion de infeccion por nueva influenza A (H1N1) en Chile al 11 de Junio de 2009 [Situation report for infection due to novel Influenza A (H1N1) in Chile June 11, 2009]. www.pandemia.cl Date last accessed: December 6, 2009. Date last update: June 11, 2009.

15 Fraser C, Riley S, Anderson RM, et al. Factors that make an infectious disease outbreak controllable. Proc Natl Acad Sci USA 2004; 101: 6146-6151.

16 World Health Organization. Morbid obesity in a developing country: the Chilean experience. www.who.int/bulletin/volumes/ 86/10/07-048785/en/ Date last accessed: December 31, 2009. Date last updated: October 2008

17 Centers for Disease Control and Prevention. Obesity and Overweight. www.cdc.gov/nchs/fastats/overwt.htm Date last accessed: December 31, 2009. Date last updated: 2008.

18 Morens DM, Taubenberger JK, Fauci AS. Predominant role of bacterial pneumonia as a cause of death in pandemic influenza: implications for pandemic influenza preparedness. I Infect Dis 2008; 198: 962-970. 